\title{
African Americans' views on research and the Tuskegee Syphilis Study
}

\author{
Vicki S. Freimuth ${ }^{\mathrm{a}}$, Sandra Crouse Quinn ${ }^{\mathrm{b}, *}$, Stephen B. Thomas ${ }^{\mathrm{c}}$, Galen Cole ${ }^{\mathrm{a}}$, \\ Eric Zook ${ }^{\mathrm{d}}$, Ted Duncan ${ }^{\mathrm{a}}$ \\ ${ }^{\text {a }}$ Centers for Disease Control and Prevention, Atlanta, GA, USA \\ ${ }^{\mathrm{b}}$ Department of Health Behavior and Health Education, School of Public Health, Rosenau Hall, CB $\sharp 400$, University of North Carolina \\ at Chapel Hill, Chapel Hill, NC 27599-7400, USA \\ ${ }^{\mathrm{c}}$ Rollins School of Public Health, Emory University, Atlanta, GA, USA \\ ${ }^{\mathrm{d}}$ Porter Novelli Inc, Washington DC, USA
}

\begin{abstract}
The participation of African Americans in clinical and public health research is essential. However, for a multitude of reasons, participation is low in many research studies. This article reviews the literature that substantiates barriers to participation and the legacy of past abuses of human subjects through research. The article then reports the results of seven focus groups with 60 African Americans in Los Angeles, Chicago, Washington, DC, and Atlanta during the winter of 1997. In order to improve recruitment and retention in research, the focus group study examined knowledge of and attitudes toward medical research, knowledge of the Tuskegee Syphilis Study, and reactions to the Home Box Office production, Miss Evers' Boys, a fictionalized version of the Tuskegee Study, that premiered in February, 1997. The study found that accurate knowledge about research was limited; lack of understanding and trust of informed consent procedures was problematic; and distrust of researchers posed a substantial barrier to recruitment. Additionally, the study found that, in general, participants believed that research was important, but they clearly distinguished between types of research they would be willing to consider participating in and their motivations for doing so. (C) 2001 Elsevier Science Ltd. All rights reserved.
\end{abstract}

Keywords: African Americans; Clinical trials; Recruitment; Research; Tuskegee study; United States

\section{Introduction}

The participation of African Americans in clinical and public health research is essential for addressing disparities in health status. However, for many different reasons, participation is low in many research studies. The authors will first review the literature that substantiates barriers to participation and focus specifically on the legacy of the Tuskegee Syphilis Study as a prime symbol of past abuses of human subjects through

*Corresponding author. Tel.: +00-1-919-966-3915; fax: $+00-1-919-966-2921$.

E-mail address: sandra_quinn@unc.edu, squinn@sph.unc.edu (S.C. Quinn). research. The authors will then report the results of seven focus groups with 60 African Americans in Los Angeles, Chicago, Washington, DC, and Atlanta. Conducted in winter, 1997, the focus group study examined knowledge of and attitudes toward medical research, knowledge of the Tuskegee Syphilis Study, and reactions to the Home Box Office production, Miss Evers' Boys, a fictionalized version of the Tuskegee Study, that premiered in February 1997.

\section{Background}

Data on participation rates suggest that African Americans are especially difficult to recruit into 
research. For example, Arean and colleagues (1993) used advertisements in local newspapers and radio stations to recruit elderly adults into a clinical trial. While this was successful for whites, only $1 \%$ of those who responded belonged to an ethnic or racial minority group. Ballard, Nash, Raiford and Harrell (1993) reported that, as of 1989 , only $9.9 \%$ or 60 of 607 participants in a study of Alzheimer's disease were African American. Svensson (1989) examined 50 clinical trials for new medications reported in Clinical Pharmacology and Therapeutics over a 3-year period. He found that the enrollment of minorities in the trials was substantially less than their proportion in the population where the trials were located. Eastman (1996) reported that African Americans comprised only $5 \%$ of participants in cancer clinical trials. Reynolds (1996) cited a similar statistic for the National Cancer Institute's Prostate, Lung, Colorectal, and Ovarian Cancer Screening Trial with only $6 \%$ of its participants being African American.

In the era of AIDS, El-Sadr and Capps (1992) state "the under-representation of minorities among participants in HIV-related drug trials is of serious concern, and of ethical, social, and scientific importance". Many authors have documented numerous barriers to participation in research. Those barriers fall into the following categories: 1) broader health care system issues; 2) characteristics of potential participants; 3) public knowledge, perceptions, and attitudes toward researchers and research; 4) behaviors and attitudes of providers and researchers. Furthermore, numerous authors have determined that lack of knowledge of research, mistrust, fear of exploitation, and the Tuskegee Syphilis Study are significant barriers. Therefore, our study, and consequently, the literature review, focuses primarily on those issues and factors associated with them.

Knowledge about research and its purposes is an important factor in willingness of African Americans to participate in clinical trials. In a study with 8 African Americans, Roberson (1994) found that African Americans, Hispanics, and Native Americans were generally familiar with the term "experimental study", but were less familiar with the term "clinical trial". African Americans, Hispanics, and Native Americans defined the purpose of an experimental study as "a study to help people", "an experiment where people are used as guinea pigs", and a "study to learn about a disease and medication" (Roberson, 1994, p. 2689). Respondents believed that the benefits of participation were to "help others", "help find a cure", "educate families", and to "assist with medical coverage" (Roberson, 1994, p. 2689). El-Sadr and Capps (1992) suggest that the lack of knowledge about the nature, purpose and procedures of medical research is a significant barrier to recruitment that requires patience, building of trust, and a thorough educational program about the purpose of clinical trials.
Knowledge of research procedures and purposes is often linked to access to health care. Many African Americans receive primary health care in emergency rooms and receive care from several physicians, "none of whom may be knowledgeable about the available protocols nor interested enough in the patient to refer $\mathrm{him} / \mathrm{her}$ for inclusion in a clinical trial" (ShaversHornaday, Lynch, Burmeister and Torner, 1997, p. 35). Additionally, patients may seek care at neighborhood institutions, where little research is conducted (ElSadr \& Capps, 1992). While much research takes place at university medical centers, many African Americans may feel that the university medical facility "is available only to select segments of the population (e.g., white, middle class) and not to the minority populations", thus limiting their participation in studies (Welsh, Ballard, Nash, Raiford \& Harrell, 1994, p. 40).

Several authors describe researchers and physician biases as barriers to recruitment of African Americans. Despite ethical standards that would prohibit such actions, researchers may limit minority participation in clinical trials because of their beliefs that "there is greater difficulty in obtaining African American compliance with a study protocol and that they have higher attrition rates" (Shavers-Hornaday et al., 1997, p. 37). Swanson \& Ward (1995) describe researchers' biases about barriers to participation as reflections of the researchers' own prejudices against the populations of interest for the research. They include "failure to accommodate cultural and economic diversity of potential study participants, failure to recognize that restrictive studies do not fully assess safety and efficacy of new treatments or preventive interventions for all populations, claims that statistical power will be reduced if women and minorities are included, inaccurate beliefs that certain populations are not at risk for specific conditions or illnesses, and failure to establish research clinics in minority institutions" (Swanson and Ward, 1995, p. 1753). They state that researchers use a common excuse for exclusion of minorities when they describe them as "hard to reach" (p. 1753), characterizing the population as difficult as opposed to understanding the broader context in which the community lives. Researchers may be reluctant to spend limited funds on actively recruiting minorities. Another factor that may limit recruitment is the small number of minority researchers (Swanson and Ward, 1995), and limited relationships between investigators and minority health care providers and caregivers.

Central to the conduct of research in community settings is the issue of trust between the investigator, the agency and community members. In a study of 1,900 subjects in biomedical research, Kass, Sugarman, Faden, and Schoch-Spana (1996) found that trust in the respondents' physician was the critical factor determining enrollment in a study. Paradoxically, trust 
contributed to the participants' lack of attentiveness to the consent process, with many reporting their decision to participate occurring prior to reading the consent form. While Kass and colleagues (1996) did not analyze their results to examine racial differences, trust in the physician may not be present for many African Americans. For example, during testimony in 1990 before the National Commission on AIDS, Mark Smith, MD., then from Johns Hopkins University, described the African American community as "already alienated from the health care system and the government" (Thomas \& Quinn, 1991, p. 1499). Freedman (1998), in her study of 13 African American women, describes her interviewees' lack of trust in their white providers, their belief that white doctors overlook diseases prevalent in the African American community, and their concern that they may be used as guinea pigs.

Distrust of researchers by many African Americans poses a well documented, serious challenge to investigator efforts to meet the mandates that require inclusion of minorities in research set out by the National Institutes of Health (NIH), the federal agency in the US that funds a significant amount of biomedical and public health research and sets the national research agenda. Gamble (1997) asserts that the history of medical experimentation on African Americans during slavery laid the foundation of distrust. Harris, Gorelick, Samuels and Bempong (1996) confirm a "legacy of mistrust", stating:

Southern blacks became a prime source for medical school dissection experiments and autopsy specimens. Fearful of this practice, some expressed the hope to die in the summer months when dissection classes were not in session. This practice continued in the postbellum South in the form of 'night-doctors' who stole and dissected the bodies of blacks (p. 631).

Abuse of human subjects involved in research has been well documented, and modern examples include the Human Radiation Experiments conducted by the US Government, birth control studies on women of color, the Brooklyn Jewish Chronic Disease Hospital and the Willowbrook Hospital cases, among others (see the Final Report of the President's Advisory Committee for a discussion of abuses of human subjects). However, the legacy of the Tuskegee Syphilis Study has emerged as the most frequently cited contemporary event to justify African Americans' suspicion of research (Quinn, 1997; Swanson \& Ward, 1995; El-Sadr \& Capps, 1992; Thomas \& Quinn, 1991; Gamble, 1993; Hatch, Moss, Saran, Presley-Cantrell \& Mallory, 1993). The Tuskegee Syphilis Study of Untreated Syphilis in the Negro Male, 1932-1972, was the longest non-therapeutic study in medical history. Conducted by the US Public Health
Service with 399 African American men as subjects and 201 African American men as controls in Macon County, Alabama, the study followed the natural history of the disease. The participants in the study were never informed that they had the disease, and when penicillin became the standard of care, the men were prevented from receiving treatment. It is estimated that between 28 and 100 men died as a result of syphilis.

Smith believes that the Tuskegee Syphilis Study "provides validation for common suspicions about the ethical even-handedness in the medical research establishment and in the federal government, in particular, when it comes to Black people" (Thomas \& Quinn, 1991, p. 1499). Before the National Commission on AIDS, Dr. Smith stated, "[The Black] communities' perspective on medical research has a historical basis which sometimes outweighs the demonstrable integrity and commitment of individual investigators" (Thomas \& Quinn, 1991, p. 1503).

Today, many believe the Study's legacy hampers recruitment for research among African Americans. During testimony to the National Commission on AIDS, Alpha Thomas, health educator with the Dallas Urban League, stated: "So many African American people that I work with do not trust hospitals or any of the other community health care service providers because of that Tuskegee Experiment. It is like if they did it then, they will do it again" (Thomas \& Quinn, 1991, p. 1503). In an effort to recruit African American women for a research project on hormone replacement therapy, Lynda Powell, professor of Preventive Medicine at Rush-Presbyterian-St. Luke's Medical Center in Chicago, initially denied the Tuskegee Study had an impact on recruitment, describing it as old, obscure, and a study that only physicians remembered. Even prior to the premiere of Miss Evers' Boys in 1997, Powell recognized her error, stating, "Each woman [African American recruiter] related a story about Tuskegee passed on by parents and grandparents... These were educated, talented women who were deeply affected. I thought only researchers knew about it" (Condor, 1997, p. 1).

In a telephone survey of 218 African American and 203 white residents of Jefferson County, Alabama, Green, Maisiak, Wang, Britt, and Ebeling (1997) found empirical evidence of the impact of the study. Fifty-two percent of African Americans were aware of the Tuskegee Study compared to $46 \%$ of whites. Twentytwo percent of African Americans compared to $10 \%$ of whites reported that, because of the Study, they were less willing to participate in research. Green and colleagues (1997) also found that African Americans were more likely to believe that people are sometimes treated poorly in research and that African Americans, in particular, are likely to be mistreated. 
James Jones, the author of Bad Blood: The Tuskegee Syphilis Experiment - A Tragedy of Race and Medicine, the definitive published history of the study, states, "No scientific experiment inflicted more damage on the collective psyche of black Americans than the Tuskegee Study" (Jones, 1992, p. 38). In part, as a response to this harmful legacy, in 1996, at a meeting at Tuskegee University, a group of individuals formed the Tuskegee Syphilis Study Legacy Committee. In its report to the Centers for Disease Control and Prevention (CDC) in May 1996, the committee argues, "the study has become a powerful symbol for the fear of exploitation in research..." (Gamble \& Fletcher, 1996, p. 7). Gamble states, "The strengthening of safeguards and the reforms in research standards that followed the public disclosure of the abuses of the Tuskegee Syphilis Study have been insufficient to change African Americans' historically based fears of medical research" (1993, p. 37).

To address the numerous barriers, a number of researchers suggest strategies for increasing participation in research among African Americans. One area in which many researchers suggest specific strategies is the relationship between researchers and the African American community. Such strategies include: raising awareness through outreach programs, local churches and community organizations; publicity campaigns targeted to African Americans; increasing awareness about studies among doctors; using community-centered communication involving trusted individuals, and involvement and commitment of culturally-sensitive staff (Freedman, 1998; Swanson and Ward, 1995; Welsh et al., 1994; Shavers-Hornaday et al., 1997; Harris et al., 1996; El-Sadr \& Capps, 1992). Other strategies include use of incentives like financial compensation, therapeutic interventions, provision of health care, access to needed services, and provision of transportation services to facilitate participation (El-Sadr \& Capps, 1992; Welsh et al., 1994; Shavers-Hornaday et al., 1997; Swanson and Ward, 1995). Patients who have no previous conception of a clinical trial need "an extensive educational effort" about trials, sampling, responsibilities of researcher and participant, and other issues in order to understand and participate in clinical trials (El-Sadr \& Capps, 1992). Swanson and Ward (1995) suggest multiple strategies in the categories of community methods, health care provider and facilities strategies, individual and family strategies, and modifications in research protocols, procedures and trial management in order to improve recruitment of minority participants into research.

While there are numerous strategies for increasing participation, some researchers call for a better understanding of the underlying distrust and cultural context in which African Americans consider research. ShaversHornaday et al., (1997) consider distrust a major barrier to the recruitment of African Americans to clinical trials, and call for further research on attitudes, beliefs and barriers to participation among African Americans. Roberson (1994) states that research is needed as "little is know about the role of mistrust as a variable that might influence participation" (p. 2690). Similarly, Swanson and Ward (1995) call for further research to study minority communities to determine those factors that both enhance clinical trial accrual and present challenges to clinical trial recruitment (p. 756). Jones (1993) specifically challenges us to deal with the impact of the Tuskegee Syphilis Study when he says, "To the extent the Tuskegee Study continues to sow suspicions and distrust, it remains a problem that must be addressed" (p. X).

While the Tuskegee Study is not the only barrier to African Americans' participation in research, since it officially ended in October 1972, its legacy continues through legend, fiction, mass media, music, and documentary studies. For example, in 1992, ABC Prime-time Live and NOVA both produced programs about the study. In 1996, a popular television series, NY Undercover, focused on the study, linking it to the issue of AIDS as a form of genocide. In 1997, Home Box Office (HBO) premiered a movie, Miss Evers' Boys, a fictionalized account of the study based on David Feldshuh's Pulitzer nominated play. The central character in Miss Evers' Boys is based on Eunice Rivers, the African American nurse who worked on the study for more than 30 years. Eighteen percent of African American households with $\mathrm{HBO}$ watched the premiere of the movie on February 22, with an overall $8 \%$ of all 19.2 million HBO subscribing households viewing it (Personal communication, Conner, February 27, 1997). Additionally, the movie is available through video stores nationwide for home viewing.

There were two rationales that contributed to this current study. While researchers have documented numerous barriers to the recruitment of African Americans into research programs, few articles actually utilize data from African American participants to examine these issues. Therefore, this study sought to directly listen to the voices of African Americans through focus groups, particularly as the premiere of Miss Evers' Boys brought the Tuskegee Syphilis Study into the public eye again. Specifically, the study sought to understand attitudes toward research among African Americans, to assess knowledge of and beliefs about the Tuskegee Syphilis Study, to identify strategies to overcome barriers to participation, and to examine the impact of viewing Miss Evers' Boys on focus group participants' attitudes.

\section{Methods}

Since most of the published literature on barriers to participation are either literature reviews, data from very 
small samples or observations from researchers/providers, focus groups were chosen as the methodology for this study because they offered an opportunity to explore participants' responses to many of the issues identified in the literature, examine their reactions more fully, and to allow new areas of inquiry to emerge. The research design called for eight focus groups, two in each of four major regions of the United States: Mid-Atlantic (Washington DC), South (Atlanta), Midwest (Chicago), and West Coast (Los Angeles) that HBO had selected as premiere sites for Miss Evers' Boys. Local chapters of the civic groups, 100 Black Men and 100 Black Women, assisted in recruitment of participants. Additionally, in Chicago, health outreach workers from the College Park Authority, a local community based organization, recruited participants. These particular groups were selected to help with recruitment because while they all serve African Americans, the populations they reach differ by class and education, thereby providing a broader pool of potential participants. Key contact people in each organization received instructions on screening criteria and recruitment procedures. Once potential participants were identified, research assistants contacted each individual to complete a more detailed screening.

While the research aim was to recruit groups that were balanced in terms of gender and age, with socioeconomic status (SES) as the primary segmentation variable, the goal was not to recruit a representative sample. For the purpose of this study, researchers defined SES by annual household income and highest level of education attained. Researchers identified an income of $\$ 20,000$ or less as low income with low educational level defined as high school diploma/GED (general education development test) or less. Moderate income was defined as $\$ 30,000$ or above with any college or above being designated as higher educational level.

A professional African American moderator conducted all sessions. A notetaker was present at all sessions which were also audiotaped. The format for each group included a discussion, the viewing of Miss
Evers' Boys, and further discussion. All notes and tapes were transcribed verbatim. All participants provided informed consent.

\section{Data analysis}

All transcripts and notes were utilized in the analysis. Initial reading of notes and transcripts focused on identifying key themes, words and phrases that occurred in response to each question. Then, the authors examined themes across the transcripts and developed broader conceptual domains. Quotes were extracted that illustrated common responses and themes across groups.

\section{Characteristics of participants}

A total of 60 individuals participated in four moderate income and three low-income focus groups. Due to logistical difficulties, the group of moderate-income participants in Chicago failed to take place. Of these 60, 20 men and 40 women participated with a median age of 37. Participants ranged in age from 19 to 78 . Five participants had less than a high school education and another 5 had a high school diploma or its equivalent. Ten participants reported some college with 6 having attended vocational or technical school. Sixteen had a college degree, and 10 participants had a graduate degree. Twenty-four participants reported annual incomes of less than $\$ 20,000$ per year, with the majority of them reporting less than $\$ 10,000$ annual income. Six participants reported annual income between $\$ 20,000$ and $\$ 30,000$, with 10 reporting incomes between $\$ 30,000$ and $\$ 50,000$. Twelve participants reported incomes above $\$ 50,000$. Thirty-nine had previously known of the Tuskegee Study. Nine participants reported some prior participation in research. For 8 participants, there is only data on gender available (see Table 1).

Table 1

Site, income of group members, and number of participants

\begin{tabular}{llr}
\hline City & Income of group members & Number of participants \\
\hline Washington, DC & moderate & 5 \\
Washington, DC & moderate & 9 \\
Los Angeles & low & 7 \\
Los Angeles & moderate & 10 \\
Atlanta & low & 10 \\
Atlanta & moderate & 7 \\
Chicago & low & 12 \\
\hline
\end{tabular}




\section{Results}

In general, geographic region, gender and SES had no perceivable influence on participant knowledge of or feelings toward either medical research in general or the Tuskegee Syphilis Study in particular. While some participants from the moderate-income groups were somewhat more knowledgeable about research, there were no differences between groups on most issues. Participants were virtually unanimous from the outset that African Americans needed to be very cautious about when and how they interacted with the medical system or government agencies. This caution was applied to all aspects of involvement with the medical community, including treatment and initiatives designed to promote the health and well being of African Americans through appropriate preventive care and behaviors. In relation to medical research, there was consensus that African Americans should generally avoid involvement given knowledge of past abuses and the inability to be certain that abuses would not reoccur.

\section{Understanding of research}

The moderator posed the following question, "What comes to mind when I say medical research?" The common responses ranged from descriptions of types of research, research as a profit making endeavor, to unethical research studies. Numerous participants described different purposes of research such as, "It's also endeavoring to find cures for various diseases, too", as well as different types of research stating "Yeah, they use some type of procedure or medication, maybe, or cutting you open, taking out something or adding something. Something that hasn't been proven yet', and "Things like clinical trials. I mean like testing, using certain people as test cases".

Another common response linked research to financial gain with comments such as: "It's an industry onto itself"; "Making money"; and "An awful lot of that I think is driven by profit, unfortunately". A number of participants immediately responded in a negative manner. As one man said, "Uh-oh. A lot of stuff has come to light recently due to declassification of information. Every thing is done in the name of medical research. Humans can be guinea pigs as well. It makes me think "uh-oh"'. This quote illustrates a theme of suspicion mentioned in several groups, "If people knew half the things they were exposed to, knowingly or unknowingly, they would be surprised".

\section{Knowledge of research terms and procedures}

To gauge knowledge about research, the moderator asked several questions about common research terms such as confidentiality, informed consent, placebo, clinical trial, protocol and randomization. In general, level of knowledge was not very high. While many participants understood confidentiality, others had misconceptions about the term. While one participant described it as "No one sees you going in or out", another thought it meant, "The data will only be used in a positive manner'.

While a significant number of participants understood what a placebo is, others did not know the term at all. Those that responded to the question, "What is a clinical trial?" were correct in that it often involved placebos for the purpose of testing the efficacy of treatments or medications. While a few correctly defined randomization, many clearly did not understand the term, with 2 participants describing it as "not given any thought" or "no specific target group". Few understood the term protocol. One participant described protocol as, "If they did something and they're trying to cover it up".

In general, participants understood the term, informed consent, to mean that they would receive information about the research process in order for them to fully consent. However, many participants expressed strong concerns about the nature and value of the informed consent process. Many stated a belief that providing consent was the equivalent of "signing away your rights" and that the process was designed primarily to protect hospitals and doctors from any legal responsibility. As one participant stated, "If you give consent, then you don't have any legal rights". Another participant feared the evolving motivations of the researchers:

Now, once you sign that, you know there's a certain amount of license, and I think that we've seen in the past where people have these ideas that their project or their grant, or their aspirations to get a Nobel Prize, or to publish an article in the Journal of the American Medical Association and...you know, after a while they lose sight of who you are....

Participants also indicated the challenge of truly giving informed consent due to difficulty in fully understanding the complexities of research. One participant described the difficulty understanding the consent forms, "You sit there and you read it but you don't necessarily understand what you're reading when you sign the paper". One person recommended a solution to the lack of understanding of consent forms by suggesting, "And it has to be in layman's terms. Some of the ones... were in hieroglyphics". Another participant 
added, "and most people don't even read it before they sign it". One participant raised the concern that the consent form may not fully represent the risks of the research, "People don't want to bias their results, so they won't tell you all of it".

\section{Past participation in medical research}

Very few participants had ever participated in medical research. Of the 9 who had, most indicated involvement in surveys or providing samples of body fluids. Only one indicated more intensive involvement in a study that included hormone injections, computed axial tomography (CAT) scans and positron emission tomography (PET) scans. For some participants, there was confusion between research and treatment, especially if the treatment was provided at a teaching hospital. One man stated, "There's a lot of hospitals that the interns, a lot of times you go in there and they're doing a lot of experimenting". Another participant described that physician changes in treatment regimes as research, "They be experimenting on me a lot of times. They give me some pills sometimes, then they give you some other medication". Another woman echoed this concern:

Because I've taken some medicine that almost killed me, it was a cancer cure and I didn't think I had a choice and I found out after I talked to the doctor, after three treatments that almost killed me, I asked him: I don't think a mule could live through those treatments and the doctor told me some of them don't. That was his answer. So they were experimenting on me...

When one participant asked, "Is a flu shot preventative or is it research?" a woman responded, "Both. Yes, I caught the flu one week after I had one of them one year, then one year I didn't get it at all'. Clearly, the distinction between treatment, prevention and research was blurry for many participants. While several participants commented that, "We all have [participated] unknowingly", and "Sometimes you're in medical research when you don't know it", others had specific ideas about the type of research conducted without consent. When one participant suggested, "Like take a commercial for a new painkiller and we watch it and then we go out and buy it and if nothing goes wrong it works", another went on to say:

I think there is a covert way of doing medical research and then there is more honest research done out in the open. As far as the process, I don't know. Like when I first started going to school, my mother didn't allow us to get those immunizations. She was a nurse and she said it was research and it wasn't proven.

\section{Motivations of researchers}

Participants correctly reported that universities, hospitals, corporations and government agencies carry out research. They reported a variety of motivations of the researchers that ranged from positive ones such as, "I think they are trying to learn or trying to cure some of these diseases", to more skepticism that they are motivated by money as reflected in this man's comment, ". . . but the bottom line is everything is money because if they make it work on you, then by production, they'll get wealthy".

\section{Who benefits from research}

The majority of participants saw the benefits of research for the public. A few recognized that research might not immediately benefit its actual participants. One man stated, "I would like to say in the early stages, I don't think the patient benefits but now, if they keep medical research going on and they refine it ..., later on, that person might receive the benefits". One participant raises the complexity of the benefits and potential costs of research for African Americans:

...medical research in this country is second to none. It's excellent. We are all beneficiaries. It's very good and very important. We have to take a lot of this like everything that we have to do as black people, we have to go into it with these mental gymnastics. So it's not cut and dried. It's not black and white and so we have to be very wary of how we approach this system.

Several participants questioned the likelihood that African Americans will benefit from much of the research conducted in the US. This was a common sentiment: "My challenge is, being African Americans, will we have access to the findings, whatever they discover, to make us well?"

\section{Motivations for participation in research}

Despite the overwhelming predisposition against participation, participants in this study provided new insights when they identified several motivations for participation in research. These include personal motivations to help themselves or a loved one through treatment provided as part of the research (typically in 
relation to a chronic or terminal illness), altruism, and financial compensation. For a number of participants, their own experience with a life-threatening or severe chronic illness might facilitate their willingness to participate. One participant who suffered from ulcerative colitis said she would be willing if she gained, "Better relief and finding out more, then coming out with another drug that could help eliminate symptoms". Another participant mentioned a theme from several groups, "I would participate if I was dying of a disease and it was fatal and I was in the last stages". While another person echoed that same motivation, she illustrated some misunderstanding of the process of randomization in research when she stated:

I was going to say if I was a victim of maybe a longterm illness and there were no other options or alternatives for me but I would like to know that I am on the more humane aspect of it. And what I mean by that is that I have a choice and I'm not selected for the one that doesn't receive any type of treatment.

A number of participants saw monetary reimbursement as a significant incentive. Another participant expressed the importance of research for all. One woman said she would be willing to participate "To find whatever diseases that are occurring, to find out the solution to that, or cure". One man illustrated the value of research in blunt terms, "Because if nobody do it, somebody gonna die, I mean, more people gonna die".

\section{Willingness to participate in research}

Participants in the focus groups identified several factors that would affect their actual willingness to participate in research. One person indicated a concern about privacy and the use of data, "Privacy. I want to try to maintain as much of my privacy as possible. You know, how much confidentiality will I really receive? What will be done with my information?" Another person expressed a common issue when he stated, "What would make me participate is being made aware before I commit to it of what I'm actually gonna be asked to do". Finally, one person raised the importance of trust in his primary provider as a critical factor in his willingness to participate in research: "I would think that my primary provider would have to endorse the research. He would have to put an endorsement on what they were doing because at this stage, I trust my doctor".

There was a wide range of opinions on willingness to participate in research with some participants indicating absolute unwillingness and others considering some types of research. However, one critical finding from the focus groups was participants' distinctions between types of research in which they would be willing to participate. Participants were most willing to consider participation in non-invasive research such as completing a survey, participating in a group discussion or allowing assessments of their health behaviors. One participant reported, "I do all kinds of surveys, I'm a survey person", and another responded that he would be willing to have researchers "asking questions about my past medical history". However, research focusing on individual behaviors was criticized for its implication that behaviors of African Americans were the primary cause of health problems.

Other participants reported willingness to participate if they perceived the risk to be low. For example, one person echoed a sentiment expressed by several when she said, "If they want something I excrete, that's fine". Many expressed concerns over adverse consequences to their own health; one man's comment sums it up, "No side effects, no side effects, no life threatening, it's not life threatening or side effects". Unwillingness increased significantly in relation to invasive procedures such as injections or ingestion of experimental medications. Many participants perceived such procedures as extremely high risk due to the inability of participants to know for sure what they were receiving and what negative effects it could have on them. One participant summed up a common reaction, "I might do it if it's research where they're taking something like fluid samples from me. But if it's injecting anything, no". Another participant stated a frequent concern, " . . they could be lying about what they're doing with the needle".

Certainly, the Tuskegee Syphilis Study provided adequate justification for many who expressed unwillingness to participate in research. One man observed, "I have a friend that went through some medical research ... but me, myself, I've always been skeptical about it because of this Tuskegee incident". That distrust was evident in numerous responses across the groups; one participant spoke passionately, "I feel like all diseases were created by research anyway, so why should I participate and help them make more?"

\section{Knowledge and perceptions of the Tuskegee Syphilis Study}

The majority of participants were familiar with the Tuskegee Study, though most lacked specific and correct information about the exact nature, extent and length of the study. All knew that it involved a study of African American men who had syphilis and did not receive treatment. One woman gave an emotional account:

They promised them things that they had no intention of delivering while they got sick and [when] 
they told the people conducting it that they were getting sick and they [researchers] were like, we'll take care of it but they had no intention because this is what they wanted to see, how it affected the human body so they let it go and it was horrible.

A significant number of participants mistakenly believed the men in the study had been purposely injected with syphilis, echoed here in one man's comment, "I heard people were given syphilis, that they were injected with it, to see what would happen". Others held different misconceptions about the study ranging from confusion that prisoners were involved to one man's comment, "... they killed the whole town, the whole town died behind that thing".

While most participants who knew about the study reported learning it from a PBS special, magazine articles, and an episode of "New York Undercover", one reported reading Jones' book, Bad Blood, and another learned about it through an AIDS class.

There was fairly widespread belief that the Tuskegee Syphilis Study was typical of other research projects, particularly when African Americans were involved. Comments included: "It was typical of the way that blacks, especially men, were exploited", and "Well, this is clearly racism... it was perceived that black people were of less value and... this kind of experiment was okay to do on black people. If these were white men... it never would have happened". From one woman's childhood, she reported, "My mother was from North Carolina. When she was a teenager, they had student doctors to kidnap certain people and she was almost kidnapped in one of these incidents".

Several participants expressed anger that they had never been taught more about the Tuskegee Study, and saw the lack of detailed information as a significant factor in the misinformation and rumors about government conspiracies and genocide. One participant linked the lack of information on the study with the creation of myths and rumors:

It is interesting that this happened relatively recently but we don't have the complete information. And it's not our fault. We go on what we heard and if we don't have the complete facts, you can imagine what we'll think. That is how the rumor mill gets started. If we are educated on it, and this film could educate us. It is a footnote in history. But if we don't have the full information, we will fill in the blanks.

Many participants identified ongoing "experiments" and "conspiracies" that they likened to the study, including Agent Orange (an herbicide used in the Vietnam War that has been associated with health outcomes), the measles vaccine study in Los Angeles in the 1990's, and immunizations. Myths persist despite the facts about such studies. For example, in the measles study referred to by several participants, the vaccine was an investigational drug approved by the US Food and Drug Administration, and there were no fatalities from its administration. However, this quote reflects a common concern:

One of the most famous studies that has just been done in the last 2 or 3 years is the measles study where the measles vaccine was not approved by the government that they were using on black babies and babies in Haiti and the islands and here in the United States, even here at - where those kids died.

Many participants view HIV/AIDS as an example of an experiment deliberately introduced to harm people of African descent. One woman reported, "Guinea pigs. I have a strong belief that syphilis and AIDS originated from a laboratory experiment. That's what they used people for". Others echoed these sentiments in different cities:

I think it is still going on now. Like AIDS, it was man-made but it kind of got out of hand. Like the flu, chicken pox and polio. It's still going on. And then the HIV in Africa and Haiti, Uganda and these countries where it's very high. Also, again, on 60 Minutes, they had where the doctors said that the batch of polio vaccine was tainted but it was too late to stop it and so these countries don't sue, you know, they just die and nobody cares in the medical field.

Several participants did indicate a belief that the Tuskegee Study represented the way things "used to be" and that some improvements had been made. However, virtually none of the participants could specifically identify steps that have been taken to improve the protection of human subjects.

\section{Reactions to Miss Evers' boys}

Following their viewing of the movie, participants expressed mixed reactions. Many participants were concerned about the emphasis on African American involvement in the study to the exclusion of the white doctors from the US Public Health Service. A number of participants were angered by Miss Evers' actions and could not understand how she continued to participate. Others countered that the racism and sexism prevalent at that time should be considered, and some questioned the way the movie focused attention on her role rather than the role of the government. Several individuals found the movie to provide simply one more indication that, "You can't trust the [profanity] white man". A small number also criticized the way the movie 
emphasized traditional black stereotypes of "naiveté" and "ignorance". Many were upset with the extremely small and meaningless compensation provided to the participants.

A significant number of viewers still questioned whether the men were deliberately injected with syphilis as illustrated by this participant's comment, "Is it possible they injected those men with syphilis? I mean it was a government study so the government was policing themselves so who's to say they didn't inject those men with syphilis?" For many viewers, it confirmed their distrust of the government as in this man's comment, "My reaction to the movie, it just confirmed my belief about the conspiracy in the United States against black people". A number of other viewers were skeptical of research and treatment initiatives. One man stated, "And this thing right here, about the deception that's going on. Today I sit here and wonder, did they really plant the viruses or the diseases that's going on now?", while a woman came back to an issue raised in many groups, "Oh, it just goes to show you that if you get like a pneumonia shot, you just don't know what you're getting". The deception of the men in the study was particularly troublesome for many participants. As one woman stated, "What disturbed me was the lie, the lie that was told from the beginning about the study".

In addition to the concern that the movie downplayed the role of the government, participants questioned why whites were not included in the study since they presumably had syphilis too, they wanted to know more about the families of the men involved and how they were affected, and were appalled that no one was held accountable. Overall, despite a variety of concerns about the factual accuracy of the movie, there was a general consensus that it provided enough information to understand what had happened at Tuskegee.

Few participants were aware of any specific ethical safeguards that were implemented following the disclosure of the Tuskegee Study. Nonetheless, there was concern about how effective such requirements would be since it would amount to the government attempting to police itself. Few perceived that available legal options would be effective, particularly if one had provided informed consent.

Watching the movie stimulated many to link the unethical treatment of the men in the study with a number of incidents of actual or perceived mistreatment by the federal government. One woman reported, "Like the most recent here in L.A. with the crack cocaine being brought in by the government. This is the latest one of the investigations, so it's like, we just don't trust, it's not trusting the government any more". Another raised concerns about Agent Orange, "I have relatives who are going through this stuff with Agent Orange. What made me feel so bad was that the government let them go so long before they started trying to do something about it". One participant linked the Tuskegee Study with today's mental health system by stating, "You know it is continuing today with mental health clinics in black neighborhoods. They are giving them drugs to keep them crazy so they can continue to have business. Same things as in the Tuskegee Study".

Finally, several participants drew larger conclusions about the relationship between African Americans, the government, and American society in general. One woman raised genocide as her concern, “...that's the overall government but I still feel that we have to charge genocide, you know, we, as a people, are still being destroyed... because everything that's being done in America is to eliminate the people of color".

Finally, one participant saw the movie as an explanation about the dramatic differences in perceptions about the trial of O.J. Simpson for murder, "The movie really explains the O.J. Simpson phenomena. It underscores the reasoning behind many black people's distrust of institutions. Hearing things like this adds fuel to the fire. It is a fundamental distrust of institutions which makes it easier to believe things like the CIA brought crack into our neighborhood".

\section{Race, trust and research}

Distrust of white researchers was a common theme. Some saw racism as the motivation behind mistreatment in research as illustrated in this woman's comment, "But the white man always has the idea that we're so inferior that we're like animals so why not kill them; why didn't they pick the white man and do it to him, give him the syphilis thing". One man articulated the linkage between race and trust when he said:

I think that most of the people who are in control of research don't look like me, and I don't have confidence in how they perceive my value and my worth. I would be very reluctant to give anybody a blank check with respect to experimenting with my body and my life, my health.

While many participants documented the events and reasons for distrust, some suggested this must be remedied. One participant stated, "So all this mistrust got to be corrected at some point if the black community is going to participate actively in any kind of ongoing research". Another participant suggested that, for her to trust researchers, she would "need to make sure that white people are also in the study". Finally, one asked for understanding of the depth of the mistrust, “...when you talk about blacks and some of our concerns you will see they're not paranoid, that there is some foundation...". 
One man offered an example of how researchers could increase trust, "I feel that if there needs to be some research done, they [researchers] should be willing to offer themselves in medical research. As a participant". However, a woman's statement illustrated some of the challenges facing researchers when potential participants may not fully understand research, "If I can have a say so or a choice in or some options, yeah. Understand all there is and then choose the option that I want".

\section{Discussion}

It is important to recognize that despite differences between focus groups on levels of education and income, many common perceptions and issues arose. While certainly results of focus groups are not generalizable to the population, the results suggest that researchers must take a number of active approaches to increase knowledge, improve communication and strengthen trust in order to enhance recruitment and retention of African Americans in research. Our findings suggest a limited understanding of research, and the presence of misconceptions that might have an impact on participation. However, this study also finds that participants, in general, recognize the value of research, and make distinctions between the types of research they would find acceptable for their own participation.

Enhancing potential participants' understanding of informed consent and research procedures is a clear mandate from the participants in this study. Providing complete information in terms that are clearly understood by potential participants is critical. Conducting formative research with potential subjects and community members prior to beginning recruitment efforts will assist in determining levels of literacy and innovative ways in which information on studies can be presented. Pretesting all consent materials and utilizing readability programs will facilitate the development of consent materials whose language is appropriate for the audience.

One crucial first step is to develop a comprehensive communication campaign about research. To facilitate motivation and participation in different types of research, the public must clearly understand what research is, its terms and procedures, and most importantly, the purposes of research. Specifically, communication about research and its purposes must deal with many respondents' misconception of potential personal benefits from participation in research. Communication to promote participation in research must appeal to the self-interest of those in the target population while, at the same time, truthfully acknowledging that research is not always without risk for those who participate. While some participants in clinical trials may benefit, it must be clear to them that the production of scientific knowledge is the primary purpose of clinical research.

Increasing trust between African Americans and researchers is a serious concern. To facilitate this, it is critically important for researchers to provide factual accounts of the Tuskegee Study and other research controversies to the public and potential participants in order to avoid development of myths that will further hamper recruitment. Researchers must be proactive in addressing scientific misconduct. Certainly, the media plays a powerful role in highlighting such misconduct, and it behooves researchers to work closely with media to provide accurate accounts of research protocols and current protections for human subjects. While Miss Evers' Boys' is a fictionalized account, it remains one example of a media portrayal of research that influences public perceptions of research. It is clearly not in the best interests of researchers to gloss over such accounts or to downplay their resonance with African Americans. Honest disclosure and dialogue provide a strong foundation for development of trust.

Furthermore, while many professionals downplay the link between instances of true scientific misconduct and conspiracy theories, participants made a direct link between lack of information about real research abuses and the development of rumors and conspiracy theory. Therefore, it is necessary to acknowledge that many African Americans view research within the context of contemporary racism, urban legends and mistrust in health care and the larger society, and that their concerns and fears have a legitimate basis in historical reality. While this broader societal issue of mistrust is critical, it is beyond the scope of researchers to address alone. However, working towards developing more trusting relationships within the context of research can potentially advance that issue within public health and health care in general. This is a critical step in beginning to address disparities in health status experienced by African Americans.

Incentives are one strategy used to enhance recruitment into research. While some of the literature advocated the use of incentives (El-Sadr \& Capps, 1992; Welsh et al., 1994; Shavers-Hornaday et al., 1997), and certainly many respondents stated that incentives such as financial compensation and access to care would impact their willingness to participate, we must urge some caution on this strategy for enhancing recruitment and retention. There is the danger particularly for very low-income individuals without access to good care, that such incentives could prove to be unintentionally coercive.

While many discuss the importance of African American investigators and research staff as a means to recruit more African Americans, this alone is not sufficient to increase trust. The class and education gap of investigators can still exist, and certainly the 
participation of African American researchers and health professionals in the Tuskegee Study did not insure that misconduct would not occur. One area in which to seek change in the capacity of researchers to work effectively with communities is in the Schools of Medicine and Public Health where future researchers are being trained. Professional preparation programs must actively address issues of culture, race and class, create opportunities for students to work with populations that differ from the student by race, ethnicity or class, and aggressively teach ethics to enhance professional skills that can reduce distrust.

The voices of participants in this study speak strongly of suspicion and distrust. As Dr. David Satcher, former Director of CDC and current Assistant Secretary of Health and Human Services and Surgeon General of the US, stated, "The distrust is hurting us. I think we've got to really focus on it" (Trafford, 1997, p. a19). The results of this study offer suggestions to overcome this distrust and to increase knowledge of research that ultimately may enhance participation by African Americans in necessary clinical and public health research, improve access to care, and impact the disparities that influence the quality of life for many African Americans.

\section{Acknowledgements}

The authors would like to acknowledge Anna Dixon for her skillful moderation of all sessions, Catherine Harbour and Ann Marie Lee for their editorial assistance, and the reviewers for their thoughtful comments.

\section{References}

Arean, P., Perri, M., Nezu, A., Schein, R., Christopher, F., \& Joseph, T. (1993). Comparative effectiveness of social problem-solving therapy and reminiscence therapy as treatments for depression in older adults. Journal of Consulting and Clinical Psychology, 61, 1003-1010.

Ballard, E., Nash, F., Raiford, K., \& Harrell, L. (1993). Recruitment of Black Elderly for Clinical Research Studies of Dementia: The CERAD Experience. The Gerontologist, $33,561-565$.

Condor, B. (June 8, 1997). Rx for women's health: FDA comes to town with a campaign to teach us how medicines affect our bodies. The Chicago Tribune, Womanews, 1.

Eastman, P. (1996). NCI hopes to spur minority enrollment in prevention and screening trials. Journal of the National Cancer Institute, 88, 236-237.

El-Sadr, W., \& Capps, L. (1992). The challenge of minority recruitment in clinical trials for AIDS. Journal of the American Medical Association, 267, 954-957.

Freedman, T. (1998). "Why Don't They Come to Pike Street and Ask Us?": Black American Women's Health Concerns. Social Science and Medicine, 47, 941-947.
Gamble, V. (1997). Under the shadow of Tuskegee: African Americans and health care. American Journal of Public Health, 87, 1773-1778.

Gamble, V., \& Fletcher, J. (1996). Final report of the Tuskegee Syphilis Study Legacy Committee. Atlanta, GA: Centers for Disease Control and Prevention.

Gamble, V. (1993). A legacy of distrust: African Americans and medical research. American Journal of Preventive Medicine, Supplement 9, 35-38.

Green, B., Maisiak, R., Wang, M., Britt, M., \& Ebeling, N. (1997). Participation in health education, health promotion, and health research by African Americans: effects of the Tuskegee syphilis experiment. Journal of Health Education, 28, 196-201.

Harris, Y., Gorelick, P. H., Samuels, P., \& Bempong, I. (1996). Why African Americans may not be participating in clinical trials. Journal of the National Medical Association, 88, 630634.

Hatch, J., Moss, N., Saran, A., Presley-Cantrell, L. \& Mallory, C. (1993). Community research: Partnership with black communities. In: D Rowley, H Tosteson (Eds.), Racial Differences in Preterm Delivery: Developing A New Research Paradigm, American Journal of Preventive Medicine, Supplement 9(6), 27-31.

Jones, J. (1993). Bad Blood: The Tuskegee Syphilis Experiment (2nd ed.). New York: Free Press.

Jones, J. (1992). The Tuskegee legacy: AIDS and the black community. Hastings Center Report, 6, 38-40.

Kass, N., Sugarman, J., Faden, R., \& Schoch-Spana, M. (1996). Trust: the fragile foundation of contemporary biomedical research. Hastings Center Report, 26, 25-29.

Quinn, S. (1997). Belief in AIDS as a form of genocide: implications for HIV prevention programs for African Americans. Journal of Health Education, 28, s6-s11.

Reynolds, T. (1996). Screening and Prevention Trials Step Up Minority Recruitment. Journal of the National Cancer Institute, 88, 1265-1266.

Roberson, N. (1994). Clinical trial participation: viewpoints from racial/ethnic groups. Cancer Supplement, 74, 26872691.

Shavers-Hornaday, V., Lynch, C., Burmeister, L., \& Torner, J. (1997). Why are African Americans under-represented in medical research studies? Impediments to participation. Ethnicity and Health, 2, 31-45.

Swanson, G., \& Ward, A. (1995). Recruiting minorities into clinical trials: Toward a participant-friendly system. Journal of the National Cancer Institute, 87, 1747-1759.

Svensson, C. (1989). Representation of American blacks in clinical trials of new drugs. Journal of the American Medical Association, 261, 263-265.

Thomas, S., \& Quinn, S. (1991). The Tuskegee syphilis study, 1932 to 1972: Implications for HIV education and AIDS risk education programs in the black community. American Journal of Public Health, 81, 1498-1505.

Trafford, A. (May 6, 1997). The ghost of Tuskegee. The Washington Post, A19.

Welsh, K. A., Ballard, E., Nash, F., Raiford, K., \& Harrell, L. (1994). Issues affecting minority participation in research studies of Alzheimer Disease. Alzheimer Disease and Associated Disorders, 8, 38-48. 